\title{
FREELY FORMING GROUPS: TRYING TO BE RARE
}

\author{
MICHAEL BAAKE ${ }^{\varrho}$, UWE GRIMM ${ }^{2}$ and HARALD JOCKUSCH ${ }^{3}$
}

(Received 23 December, 2005; revised 22 June, 2006)

\begin{abstract}
A simple weakly frequency dependent model for the dynamics of a population with a finite number of types is proposed, based upon an advantage of being rare. In the infinite population limit, this model gives rise to a non-smooth dynamical system that reaches its globally stable equilibrium in finite time. This dynamical system is sufficiently simple to permit an explicit solution, built piecewise from solutions of the logistic equation in continuous time. It displays an interesting tree-like structure of coalescing components.

2000 Mathematics subject classification: primary 92D25, 91A40; secondary 34A36, 34A05, 34D20.

Keywords and phrases: population dynamics, non-smooth dynamical systems, stable equilibria, logistic equation.
\end{abstract}

\section{Introduction}

Imagine a large, but finite population of $N$ individuals, with $N$ fixed. Imagine further that they are divided into $M$ disjoint groups, of type $i$ and size $n_{i}>0,1 \leq i \leq M$, so that $n_{1}+\cdots+n_{M}=N$. Let us assume that there is an advantage in belonging to a smaller group, but that changing the group is only reasonable if individuals from different groups meet. As a justification for this setting, we envision that each individual might only know the size of its own group, but not necessarily the size of any other one. Consequently, a comparison requires the exchange of information. For simplicity, we assume honesty in this process.

To specify the dynamics, we assume that, at a fixed rate (which we may choose to be $\mathbf{1}$ for simplicity), two individuals chosen at random from the entire population

\footnotetext{
'Fakultät für Mathematik, Univinersität Bielefeld, Box 100131, 33501 Bielefeld, Germany; e-mail: mbaake@math.uni-bielefeld.de.

${ }^{2}$ Department of Mathematics, The Open University, Walton Hall, Milton Keynes MK7 6AA, UK;

e-mail: u.g.grimm@open.ac.uk.

${ }^{3}$ Fakultät für Biologie, Univinersität Bielefeld, Box 100131, 33501 Bielefeld, Germany; e-mail: h.jockusch@uni-bielefeld.de.

(C) Australian Mathematical Society 2006, Serial-fee code 1446-1811/06
} 
meet and act according to the following protocol. If both are from the same group, or from groups of the same size, no action is taken; otherwise, the individual from the larger group joins the smaller one, by changing type accordingly. We assume that the advantage depends on group size only, not on type. Note that a situation where $n_{j}=n_{i}+1$ is changed into $n_{i}=n_{j}+1$ is overall neutral, and also neutral from the point of view of the acting individual.

In this short note, we consider the resulting dynamics in the deterministic limit as $N \rightarrow \infty$, with a fixed number of types. This approach is appropriate because the protocol is deterministic, and no extinction can occur in the finite size situation. Therefore, no stochastic effects of relevance are possible. Consequently, we shall look at the deterministic time evolution of the corresponding (discrete) probability distribution $\left\{p_{i} \mid 1 \leq i \leq M\right\}$ of the types. This setting is known as the infinite population limit (IPL), compare [6, Chapter 11.2] for background material and [2, 10, 14] for recent examples. For a finite population, $p_{i}=n_{i} / N$ are the type frequencies. The model thus shows a frequency dependence, which is weak in the sense that only the signs of the differences $p_{i}-p_{j}$ matter, but not their magnitudes.

This approach leads to a simple, but rather interesting system of nonlinear ODEs, with discontinuous right-hand side. The latter property requires some care concerning the concepts of a solution and of an equilibrium, since the standard results of ODE theory do not directly apply. However, this will cause no problem in our case, where we shall see that the globally stable equilibrium, $p_{1}=\cdots=p_{M}=1 / M$, is reached in finite time from any initial condition in the interior of the probability simplex (see below for details).

Our simple example is illustrative in the sense that a biologically relevant, nonsmooth dynamical system can be looked at and solved explicitly, without invoking the general theory [8] or resorting to abstract results [5]. In fact, to derive the solution, one only needs a good understanding of the logistic equation in continuous time, compare [1, Chapter 1]. In our multi-dimensional system, the solution curves of the various components display a tree-like coalescing structure. The corresponding coalescing times are the relevant time scales that would also show up in smooth approximations of this non-smooth dynamical system (where they appear as the characteristic times of the exponential closing-in of the components).

The model is a toy example of game theoretic flavour, compare [11], where a key ingredient is the use of non-local information (the group sizes). As such, it can be considered as a model of population dynamics $[4,11]$, but is rather different from the standard models of population genetics, compare [3, 7]. As possible applications, one could think of population patches (spatial advantage), food specialisation (supply advantage), advertising strategies (promotional advantage) or name choosing (recognisability), to name but a few.

Similar models, and also more general ones, have been investigated in mathematical 
game theory. One early example is the ideal free distribution of Fretwell and Lucas in ecology [9], which describes the spatial distribution of a population among a fixed number of resource patches. This is related to the evolutionary stable strategies of game theory, see [4] for a detailed discussion. Various dynamics, including imitation and replicator dynamics, are considered in this context, compare the excellent review by Hofbauer and Sigmund [12] with its extensive bibliography.

Even though our model can be seen as a special case of imitation dynamics, compare [11, Section 8.1] and [12, Section 3.2], it results from a specific finite size model via the infinite population limit, and is thus fixed. Since we see this as a first step towards more realistic models including stochastic and finite-size effects, we find it slightly more natural to use the classic notions of dynamical systems rather than to formulate the model and its solution in the language of game theory (we shall give the translation below for comparison).

\section{The dynamical systems and their equilibria}

The reservoir of types, or state space, is $\{1, \ldots, M\}$, that is, there are finitely many different states. In the IPL limit $[2,6,14]$, one considers the deterministic time evolution of probability vectors $\boldsymbol{p}=\left(p_{1}, \ldots, p_{M}\right)$ with $p_{i} \geq 0$ and $\sum_{i} p_{i}=1$, where $p_{i}$ is the proportion of the (infinite) population in state $i$. For the model described above, with $p=p(t)$, this gives the system of ODEs

$$
\dot{p}_{m}=\sum_{n} p_{m} p_{n} \operatorname{sgn}\left(p_{n}-p_{m}\right)
$$

where sgn is the signum function, that is,

$$
\operatorname{sgn}(x)=\left\{\begin{aligned}
1, & x>0 \\
0, & x=0 \\
-1, & x<0
\end{aligned}\right.
$$

More generally, one could consider the ODEs

$$
\dot{p}_{m}=\sum_{n} p_{m} p_{n} \varphi\left(p_{n}-p_{m}\right)
$$

where $\varphi:[-1,1] \longrightarrow \mathbb{R}$ is an odd function (that is, $\varphi(-x)=-\varphi(x)$ ) which is continuous, or (as sgn above) piecewise continuous with only finitely many jumps. In particular, $\varphi(0)=0$.

In general, such discontinuities require solutions to be defined accordingly, for example, in the sense of Filippov [8, Section 4], thus referring to the well developed 
theory of ODEs with discontinuous right-hand sides. Fortunately, and not untypically for models with a biological context, one can introduce all ingredients required for our model in an elementary way. Moreover, one can make most results explicit, thus illustrating aspects usually obtained by qualitative theory.

Let us first assume that $\varphi$ is continuous. Then, the forward flow of the dynamical system (2.2) leaves the cone of positive vectors invariant, which follows from the observation that $p_{m}(t)=0$ implies $\dot{p}_{m}(t)=0$, together with standard arguments from [1, Chapter 16]. Since $\varphi(0)=0$, one also finds

$$
\sum_{m, n} p_{m} p_{n} \varphi\left(p_{n}-p_{m}\right)=\sum_{m<n} p_{m} p_{n}\left(\varphi\left(p_{n}-p_{m}\right)+\varphi\left(p_{m}-p_{n}\right)\right)=0,
$$

whence $\frac{d}{d t}\left(p_{1}+\cdots+p_{M}\right)=0$ and total mass is preserved. In particular, the forward flow of (2.2) preserves the simplex of probability vectors,

$$
\mathscr{P}_{M}:=\left\{p \in \mathbb{R}^{M} \mid p_{i} \geq 0 \text { and } \sum_{i} p_{i}=1\right\} .
$$

This is in line with the probabilistic interpretation chosen above.

In an appropriate generalisation, this remains true for the dynamical system (2.1) with its discontinuous right-hand side. In general, this claim requires some care, and is usually substantiated by viewing the ODE (2.1) within the scheme of socalled differential inclusions, where one considers equations of the form $\dot{p}_{n} \in F(t, p)$ with set-valued right-hand sides [13, Section 2], see also [12, Section 3.3] for its appearance in game theory, for example, in the context of the so-called best response dynamics. For our system (2.1), the function $\operatorname{sgn}(x)$ would simply be replaced by the set-valued function $\operatorname{Sgn}$, defined by $\operatorname{Sgn}(x)=\{\operatorname{sgn}(x)\}$ for $x \neq 0$ together with $\operatorname{Sgn}(0)=[-1,1]$, compare [13, Example 2.1.1]. Since our system is autonomous and well-behaved, it is then essentially straight-forward, following [13, Theorems 2.2.1 and 2.2.2], to establish the existence and uniqueness result for its solution in forward time. Fortunately, Equation (2.1) permits a much simpler approach, based directly on the single-valued function $\operatorname{sgn}(x)$.

Clearly, solutions of (2.1) can no longer be expected to be differentiable everywhere. The starting point is then the class of absolutely continuous functions, which are differentiable almost everywhere. A solution of our ODE (2.1) is then a function of this class that satisfies (2.1) almost everywhere. As we shall see explicitly below, the solutions of (2.1) are actually piecewise smooth, with only finitely many points of non-differentiability (coinciding with the coalescence points of components). More precisely, at the glueing points, the value of the previous segment provides the initial value for the next. It will always be evident that this construction yields the unique solution. 
In what follows, we shall only consider the dynamical system restricted to the probability simplex $\mathscr{P}_{M}$. Moreover, we mainly consider initial values from its interior, because otherwise, in view of the right-hand side of (2.1), we could start with a smaller state space right away, that is, with a probability simplex of smaller dimension. In this sense, the restriction to the interior of $\mathscr{P}_{M}$ covers all cases, by suitable choice of $M$.

The equilibria of our systems (2.1) or (2.2) certainly include the uniform distribution on $\{1, \ldots, M\}$ or that on any subset of it. These correspond to the centre of the simplex $\mathscr{P}_{M}$ or to the centre of some boundary of it, the latter effectively being a simplex $\mathscr{P}_{M^{\prime}}$ with $M^{\prime}<M$. Further equilibria can exist if $\varphi(x)$ has zeros other than $x=0$. However, we are primarily interested in stable equilibria, such as $p_{i} \equiv 1 / M$ for (2.1). In fact, the latter is even globally stable, meaning that any initial condition from the interior of $\mathscr{P}_{M}$ will converge, under the forward flow, to this equilibrium, compare [12, Section 2.2] for a brief discussion of these concepts.

Let us briefly expand on this point. Observing that both models (2.1) and (2.2) can be viewed as special cases of imitation dynamics in game theory (with the negative identity matrix being the payoff matrix, compare [11, Eq. 8.3]), one can derive that they possess a Lyapunov function, subject to certain conditions on the function $\varphi$. This can also be seen explicitly as follows. If we define $f(t):=\sum_{m}\left(p_{m}(t)\right)^{2}$, a simple double summation argument using (2.2) shows that

$$
\dot{f}=-\sum_{m, n} p_{m} p_{n}\left(p_{n}-p_{m}\right) \varphi\left(p_{n}-p_{m}\right)
$$

Consequently, as all $p_{m} \geq 0, \dot{f}(t) \leq 0$ if $\varphi$ is a non-decreasing odd function (note that $x \varphi(x)$ is then even and $\geq 0$ ).

If $x=0$ is the only zero of $\varphi(x)$ and $p(t)$ a point in the interior of $\mathscr{P}_{M}$, one has $\dot{f}(t)<0$ unless $p(t)$ is the centre of $\mathscr{P}_{M}$. This establishes $f$ as a strict Lyapunov function for the interior of $\mathscr{P}_{M}$ in these cases, see [1] for details on this concept. This observation establishes the global stability result of the equilibrium point $p_{1}=\cdots=$ $p_{M}=1 / M$, both for (2.1) and (2.2). The hierarchy of boundaries of $\mathscr{P}_{M}$ can be treated separately, by identifying them with appropriate cases of smaller dimension.

\section{Solution of the case $M=2$}

Let us consider Equation (2.1) for $M=2$. Setting $p_{1}=p$ and $p_{2}=q=1-p$, it is clear that it now suffices to consider the single ODE

$$
\dot{p}=p(1-p) \operatorname{sgn}(1-2 p) .
$$


If $p(t)<1 / 2$, this simplifies to $\dot{p}=p(1-p)$, which is the well-known logistic equation in continuous time, compare [1, Chapter 1]. It has the solution

$$
p(t)=\frac{1}{1+\alpha e^{\left(t_{0}-t\right)}} \quad \text { with } \quad \alpha=\frac{1-p\left(t_{0}\right)}{p\left(t_{0}\right)} .
$$

If the initial condition of $(3.1)$ is $0<p\left(t_{0}\right)<1 / 2$, this formula also provides the solution we need, until $p(t)$ reaches the value $1 / 2$, whereupon the solution continues as $p(t) \equiv 1 / 2$ - the globally stable equilibrium. As is not untypical of ODEs with discontinuous right-hand sides, compare [8, Section 15], this happens after a finite time, namely at

$$
t_{1}=t_{0}+\log \frac{1-p\left(t_{0}\right)}{p\left(t_{0}\right)}=t_{0}+\log (\alpha) .
$$

Similarly, if $p(t)>1 / 2$, Equation (3.1) simplifies to $\dot{p}=-p(1-p)$. Provided that $1>p\left(t_{0}\right)>1 / 2$, the solution now is

$$
p(t)=\frac{1}{1+\alpha e^{\left(t-t_{0}\right)}},
$$

with $\alpha$ as before, until $p(t)$ hits $1 / 2$ from above. This happens at time

$$
t_{2}=t_{0}+\log \frac{p\left(t_{0}\right)}{1-p\left(t_{0}\right)}=t_{0}-\log (\alpha) \text {. }
$$

In this rather simple case, $q=1-p$, and the complete solution is thus given in closed form, with one coalescence point at time $t_{1}$ or $t_{2}$, depending on the initial condition. If $\alpha=1$ (that is, $p\left(t_{0}\right)=1 / 2$ ), $t_{1}=t_{2}=t_{0}$, and one is in the equilibrium from the very beginning.

\section{Some comments on the general structure}

If $M>2$, one can simplify the situation enormously by assuming, without loss of generality, that the initial condition is non-degenerate (meaning that all types occur) and ordered, so that

$$
0<p_{1} \leq p_{2} \leq \cdots \leq p_{M}<1 .
$$

If the $p_{i}$ are all equal, we must have $p_{i} \equiv 1 / M$, that is, we are (and stay) in equilibrium. So, let us assume that at least one further inequality sign is proper, and that the leftmost position where this happens is between $\ell$ and $\ell+1$. In other words,

$$
0<p_{1}=\cdots=p_{\ell}<p_{\ell+1} \leq \cdots \leq p_{M}<1 .
$$



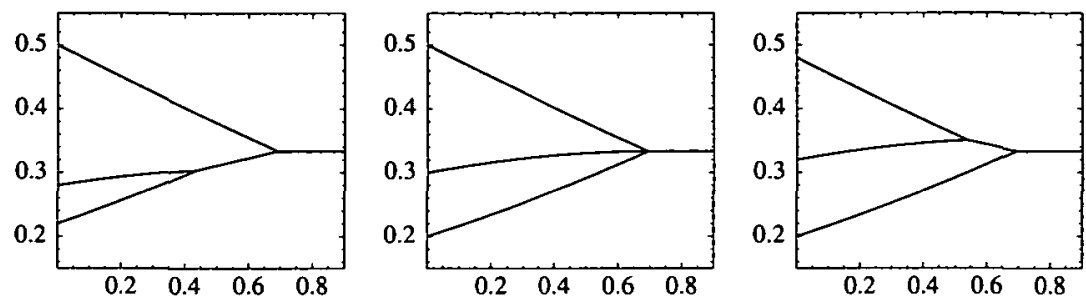

FIGURE 1. Examples of the non-degenerate classes of solutions for $M=3$. The solutions $\left(p_{1}(t), p_{2}(t), p_{3}(t)\right)$ are drawn on top of each other, against time on the horizontal axis. Initial conditions at $t_{0}=0$ are, from left to right, $(0.22,0.28,0.5),(0.2,0.3,0.5)$ and $(0.2,0.32,0.48)$. The equilibrium $(1 / 3,1 / 3,1 / 3)$ is reached at $t=\log (2)$ in all three cases. In the left (right) frame, the first coalescence happens at $t=\log (17 / 11)$ (at $t=\log (12 / 7))$.

Since $\sum_{j} p_{j}=1$, one then has $\sum_{j=\ell+1}^{M} p_{j}=1-\ell p_{i}$, for any $1 \leq i \leq \ell$. Then, a short calculation with Equation (2.1) shows that the time evolution of all $p_{i}$ with $1 \leq i \leq \ell$ is identical, and given by the ODE

$$
\dot{p}=p(1-\ell p),
$$

as long as $p_{\ell}<p_{\ell+1}$ remains true. This variant of the logistic equation has the solution

$$
p(t)=\frac{1}{\ell+\beta e^{\left(t_{0}-t\right)}} \quad \text { with } \quad \beta=\frac{1}{p\left(t_{0}\right)}-\ell .
$$

In general, the situation can be described as follows. Whatever the initial condition is, one can bring it to the standard form (4.1), by a permutation of the types. In forward time, the ordering then remains that way. Step by step, two or more neighbouring $p_{i}$ coalesce, all in finite time, until the equilibrium is reached (also in finite time). With increasing $M$, there is a growing number of different possibilities for the emerging coalescent trees of components (not to be mixed up with the result of the well-known coalescent process from population genetics, compare $[3,7])$, which makes a closed formula for the solution cumbersome. The general picture, however, is rather intuitive, and will become evident from the explicit solution for $M=3$.

\section{Solution of the case $M=3$}

Let us consider our system (2.1) for $M=3$ and assume $0<p_{1}<p_{2}<p_{3}<1$ in order to exclude any degenerate situation. It is clear that we now have three different coalescent trees to distinguish, see Figure 1.

The system of ODEs now reads

$$
\dot{p}_{1}=p_{1}\left(1-p_{1}\right), \quad \dot{p}_{2}=p_{2}\left(p_{3}-p_{1}\right), \quad \dot{p}_{3}=-p_{3}\left(1-p_{3}\right) .
$$


With $\alpha_{i}=\left(1-p_{i}\left(t_{0}\right)\right) / p_{i}\left(t_{0}\right)$, the solutions $p_{1}$ and $p_{3}$ start as

$$
p_{1}(t)=\frac{1}{1+\alpha_{1} e^{\left(t_{0}-t\right)}}, \quad p_{3}(t)=\frac{1}{1+\alpha_{3} e^{\left(t-t_{0}\right)}}
$$

while the solution for $p_{2}$ can be obtained from here by insertion and elementary integration,

$$
p_{2}(t)=p_{2}\left(t_{0}\right) \frac{\left(1+\alpha_{1}\right)\left(1+\alpha_{3}\right)}{\left(1+\alpha_{1} e^{\left(t_{0}-t\right)}\right)\left(1+\alpha_{3} e^{\left(t-t_{0}\right)}\right)} .
$$

These solutions remain valid until $p_{1}$ meets with $p_{2}$ or $p_{2}$ with $p_{3}$ (whatever is first), or both (if they coincide). A simple calculation gives the coalescing times

$$
t_{12}=t_{0}+\log \left(1+\frac{p_{2}-p_{1}}{p_{1}\left(p_{1}+p_{2}\right)}\right) \quad \text { and } \quad t_{23}=t_{0}-\log \left(1-\frac{p_{3}-p_{2}}{p_{3}\left(p_{2}+p_{3}\right)}\right) \text {, }
$$

where the $p_{i}$ are to be read as $p_{i}\left(t_{0}\right)$.

If $t_{12}=t_{23}$, all three curves $p_{i}(t)$ coalesce in one point, $(1 / 3,1 / 3,1 / 3)$. From here, the solution is then $p_{i}(t) \equiv 1 / 3$, the globally stable equilibrium of this situation, see the middle example in Figure 1. This happens if and only if the initial conditions satisfy

$$
p_{2}=\frac{3 p_{1}\left(1-p_{1}\right)}{1+3 p_{1}}
$$

in addition to $p_{1}+p_{2}+p_{3}=1$.

Otherwise, we have to distinguish the cases $t_{12}<t_{23}$ and $t_{12}>t_{23}$ (which correspond to replacing equality in Equation (5.2) by $<$ or $>$, respectively). In the former case, the above solutions for $p_{1}(t)$ and $p_{2}(t)$ are valid until $t=t_{12}$. Afterwards, $p_{12}(t)=p_{1}(t)=p_{2}(t)$ satisfies Equation (4.2) with $\ell=2$, and solution

$$
p_{12}(t)=\frac{1}{2+\beta e^{\left(t_{12}-t\right)}}, \quad \text { with } \quad \beta=\frac{1}{p_{1}\left(t_{12}\right)}-2 .
$$

The solution for $p_{3}(t)$ from (5.1) remains valid until it meets with $p_{12}(t)$, which happens at

$$
t_{12 \mid 3}=t_{0}+\log \frac{2 p_{3}\left(t_{0}\right)}{1-p_{3}\left(t_{0}\right)} .
$$

This time can easily be calculated from the condition $p_{3}\left(t_{12 \mid 3}\right)=1 / 3$.

In the remaining case, $t_{12}>t_{23}$, we have

$$
p_{23}(t)=\frac{1}{2+\beta e^{\left(t-t_{23}\right)}}, \quad \text { with } \quad \beta=\frac{1}{p_{3}\left(t_{23}\right)}-2 \text {, }
$$


after $p_{2}(t)$ and $p_{3}(t)$ met at $t=t_{23}$, until $p_{23}(t)$ meets with $p_{1}(t)$ given in Equation (5.1) at time

$$
t_{1 \mid 23}=t_{0}+\log \frac{1-p_{1}\left(t_{0}\right)}{2 p_{1}\left(t_{0}\right)},
$$

obtained from the condition $p_{1}\left(t_{1 \mid 23}\right)=1 / 3$. Figure 1 shows a representative example, from which the general situation is clear.

\section{Concluding remarks}

Equilibria of smooth dynamical systems are usually reached only asymptotically. The above toy model does so in finite time, which is a consequence of the discontinuous right-hand side of (2.1). If the latter were replaced by a smooth approximation (for example, a sigmoid curve), neighbouring lines would no longer meet in finite time, but close in exponentially fast instead - otherwise, the qualitative picture remains the same. In particular, if (2.2) is considered with an odd function $\varphi$ that is strictly monotonically increasing (hence including sigmoid curves), the nature of the globally stable equilibrium remains unchanged. An advantage of the discontinuous system is its exact solvability, including the calculation of all relevant time scales.

As to the relevance of our findings for large, but finite systems, fluctuations can lead to violations of the order relation (4.1). However, it is clear that the convergence to the equilibrium qualitatively remains the same if $N$ is a multiple of $M$. Otherwise, the equilibrium is reached up to fluctuations of at most one individual per group, that is, of order $1 / N$. This mirrors the deviations from the expectation values (over all realisations of the stochastic process), the latter coinciding with the equilibrium of the IPL limit in this simple case.

It might be interesting to extend the above model by means of a stochastic (rather than deterministic) protocol, and to compare it with related models of game theory $[4,11]$. This will automatically lead to various extensions and generalisations. To reach a more complete and realistic picture, it will be necessary to develop a proper stochastic frame and to investigate relevant finite size effects by suitable simulations, see also [12] for a brief discussion of these issues. These two aspects will certainly require more attention in the future.

\section{Acknowledgements}

It is our pleasure to thank E. Baake and J. Hofbauer for helpful criticism and W.-J. Beyn for bringing reference [13] to our attention. Financial support from German Academic Exchange Service (DAAD, to M.B.), British Council (project 
ARC 1213, to U.G.) and Fonds der Chemischen Industrie (FCI, to H.J.) is gratefully acknowledged.

\section{References}

[1] H. Amann, Ordinary Differential Equations (de Gruyter, Berlin, 1990).

[2] M. Baake and E. Baake, "An exactly solved model for mutation, recombination and selection", Can. J. Math. 55 (2003) 3-41.

[3] R. Bürger, The Mathematical Theory of Selection, Recombination, and Mutation (Wiley, Chichester, 2000).

[4] R. Cressman, V. Křivan and J. Garay, "Ideal free distributions, evolutionary games, and population dynamics in multiple-species environments", Amer. Natur. 164 (2004) 473-489.

[5] K. Deimling, Multivalued Differential Equations (de Gruyter, Berlin, 1992).

[6] S. N. Ethier and T. G. Kurtz, Markov Processes - Characterization and Convergence (Wiley, New York, 1986).

[7] W. J. Ewens, Mathematical Population Genetics. I. Theoretical Introduction, 2nd ed. (Springer, New York, 2004).

[8] A. F. Filippov, Differential Equations with Discontinuous Righthand Sides (Kluwer, Dordrecht, 1988).

[9] S. D. Fretwell and H. L. Lucas, "On territorial behavior and other factors influencing habitat distribution in birds. I. Theoretical development", Acta Biotheor. 19 (1970) 16-36.

[10] T. Garske and U. Grimm, "A maximum principle for the mutation-selection equilibrium of nucleotide sequences", Bull. Math. Biol. 66 (2004) 397-421.

[11] J. Hofbauer and K. Sigmund, Evolutionary Games and Population Dynamics (Cambridge University Press, Cambridge, 1998).

[12] J. Hofbauer and K. Sigmund, "Evolutionary game dynamics", Bull. Amer. Math. Soc. (N.S.) 40 (2003) 479-519.

[13] M. Kunze, Non-Smooth Dynamical Systems, Springer, Berlin (Springer, Berlin, 2000).

[14] O. Redner and M. Baake, "Unequal crossover dynamics in discrete and continuous time", J. Math. Biol. 49 (2004) 201-226. 